\title{
Disjoint Segments with Maximum Density
}

\author{
Yen Hung Chen ${ }^{1}$, Hsueh-I Lu ${ }^{2, \star}$ and Chuan Yi Tang ${ }^{1}$ \\ 1 Department of Computer Science, \\ National Tsing Hua University, Hsinchu 300, Taiwan, R.O.C. \\ \{dr884336, cytang\}@cs.nthu.edu.tw \\ 2 Department of Computer Science and Information Engineering, \\ National Taiwan University, Taipei 106, Taiwan, R.O.C. \\ hil@csie.ntu.edu.tw
}

\begin{abstract}
Given a sequence $A$ of numbers and two positive integers $\ell$ and $k$, we study the problem to find $k$ disjoint segments of $A$, each has length at least $\ell$, such that their sum of densities is maximized. We give the first known polynomial-time algorithm for the problem: For general $k$, our algorithm runs in $O(n \ell k)$ time. For the special case with $k=2$ (respectively, $k=3$ ), we also show how to solve the problem in $O(n)$ (respectively, $O\left(n+\ell^{2}\right)$ ) time.
\end{abstract}

\section{Introduction}

Let $A=\left\langle a_{1}, a_{2}, \ldots, a_{n}\right\rangle$ be the input sequence of $n$ numbers. Let $A_{i, j}$ denote the consecutive subsequence $\left\langle a_{i}, a_{i+1}, \ldots, a_{j}\right\rangle$ of $A$. The length of $A_{i, j}$, denoted $\left|A_{i, j}\right|$, is $j-i+1$. The density of $A_{i, j}$, denoted $d\left(A_{i, j}\right)$ is $\frac{a_{i}+a_{i+1}+\cdots+a_{j}}{j-i+1}$ of $A_{i, j}$. Observe that with an $O(n)$-time preprocessing to compute all $O(n)$ prefix sums $a_{1}+a_{2}+\cdots+a_{j}$ of $A$, the density of any segment $A_{i, j}$ can be obtained in $O(1)$ time.

Two segments $A_{i, j}$ and $A_{i^{\prime}, j^{\prime}}$ of $A$ are disjoint if $i \leq j<i^{\prime} \leq j^{\prime}$ or $i^{\prime} \leq$ $j^{\prime}<i \leq j$. Two segments of $A$ overlap if they are not disjoint. Motivated by the locating GC-rich regions [9, 14, 15, 16, CpG islands [3, 5, 11, 18, in a genomic sequence and annotating multiple sequence alignments [17, Lin, Huang, Jiang and Chao 13 formulated and gave an $O(n \log k)$-time heuristic algorithm for the problem of identifying $k$ disjoint segments of $A$ with maximum sum of densities. Specifically, given two additional positive integers $k$ and $\ell$, the problem is to find $k$ disjoint segments of $A$, each has length at least $\ell$, such that the sum of their densities is maximized. We present the first known polynomial-time algorithm to solve the problem. Our algorithm runs in $O(n \ell k)$ time for general $k$. We also show that the special case with $k=2$ (respectively, $k=3$ ) can be solved in $O(n)$ (respectively, $\left.O\left(n+\ell^{2}\right)\right)$ time.

\footnotetext{
* The corresponding author. Address: 1 Roosevelt Road, Section 4, Taipei 106, Taiwan, R.O.C. Webpage: www.csie.ntu.edu.tw/ hil/.
} 
Related work. When $k=1$, the problem studied in the present paper becomes the extensively studied maximimum-density segment problem 2, 6, 9, 10, 12, The problem for general $k$ is also closely related to the GTile with bounded number of tiles problem [1, which is a natural extension of the maximum-sum segment problem studied in [12,4.

The rest of this paper is organized as follows. Section 2 describes our $O(n \ell k)$ time algorithm for general $k$. Section 3 shows how to solve the case with $k=2$ in $O(n)$ time. Section 4 shows how to solve the case with $k=3$ in $O\left(n+\ell^{2}\right)$ time. Section 5 concludes the paper with open questions.

\section{Our Algorithm for General $k$}

For a set $U$ of segments, let $D(U)=\sum_{S \in U} d(S)$. A set of segments is feasible to our problem if it consists of $k$ disjoint segments of $A$, each has length at least $\ell$. A set $U^{*}$ of segments is optimal if $U^{*}$ is feasible and $D\left(U^{*}\right) \geq D(U)$ holds for any feasible set $U$.

Lemma 1. There exists an optimal set $U^{*}$ of segments such each segment in $U^{*}$ has length less than $2 \ell$.

Proof. Suppose that $U^{*}$ contains a segment $A_{i, j}$ with $\left|A_{i, j}\right| \geq 2 \ell$. Then, both $U^{*} \cup\left\{A_{i, i+\ell-1}\right\}-\left\{A_{i, j}\right\}$ and $U^{*} \cup\left\{A_{i+\ell, j}\right\}-\left\{A_{i, j}\right\}$. Moreover, one of them has to be optimal, since $\max \left(d\left(A_{i, i+\ell-1}\right), d\left(A_{i+\ell, j}\right)\right) \geq d\left(A_{i, j}\right)$. We then use the new optimal set to replace the original $U^{*}$. The lemma is proved by continuing this process until each segment in the resulting optimal set $U^{*}$ has length less than $2 \ell$.

According to Lemma 1, it suffices to focus on segments with lengths at least $\ell$ and less than $2 \ell$. Let $\rho$ be the number of such segments in A. Clearly, $\rho=O(n \ell)$. Define $G$ to be a graph on these $\rho$ segments such that two nodes in $G$ are adjacent if and only if their corresponding segments overlap in $A$. Observe that $G$ is an interval graph. Let the weight of each node be the density of its corresponding segment. Then, the problem to compute an optimal set $U^{*}$ of segments becomes the problem to identify a maximum weight independent set of $G$ that has size $k$. To the best of our knowledge, no such an algorithm is known, although the version without restriction on the size has been studied in the literature [8,7].

Our algorithm for identifying an optimal $U^{*}$ is via the standard technique of dynamic programming as shown below. For each $j=1,2, \ldots, n$, let $A_{j}$ consist of the segments $A_{i, j}$ of $A$ with $1 \leq i \leq j \leq n$ and $\ell \leq\left|A_{i, j}\right|<2 \ell$. For each $j=1,2, \ldots, n$, let $U_{j, t}^{*}$ denote a set of $t$ disjoint segments of $A_{1, j}$, each has length at least $\ell$ and less than $2 \ell$, such that $D\left(U_{j, t}^{*}\right)$ is maximized. Note that $U^{*}=U_{n, k}^{*}$. One can easily compute all $U_{j, 1}^{*}$ with $1 \leq j \leq n$ in $O(n \ell)$ time. For technical reason, if $j<t \ell$, then let $U_{j, t}^{*}=\emptyset$ and $D\left(U_{j, t}^{*}\right)=-\infty$. To compute all $O(n k)$ entries of $U_{j, t}^{*}$ in $O(n \ell k)$ time, we use the following straightforward procedure for each $t>1$ and $j \geq t \ell$.

Let $U_{j, t}^{*}=\left\{A_{s, j}\right\} \cup U_{s-1, t-1}^{*}$, where $s$ is an index $i$ that maximizes $d\left(A_{i, j}\right)+D\left(U_{i-1, t-1}^{*}\right)$ over all indices $i$ such that $A_{i, j}$ is a segment in $A_{j}$. 
Since each $A_{j}$ has size $O(\ell)$, if those $U_{j, t-1}^{*}$ with $j=1,2, \ldots, n$ are available, then all $U_{j, t}^{*}$ with $j=1,2, \ldots, n$ can be computed in $O(n \ell)$ time. One can then obtain $U^{*} \stackrel{=}{=} U_{n, t}^{*}$ in $O(n \ell k)$ time by iterating the above process for $t=2,3, \ldots, k$. Therefore, we have the following theorem.

Theorem 1. It takes $O(n \ell k)$ time to find $k$ disjoint segments of a length- $n$ sequence, each has length at least $\ell$, such that the sum of their densities is maximized.

\section{Our Algorithm for $k=2$}

It turns out that the linear time algorithm of Chung and $\mathrm{Lu}$ [2] for the case with $k=1$ can be a useful subroutine to solve the case with $k=2$ in linear time. For each $i=1,2, \ldots, n$, let $P_{i}$ (respectively, $Q_{i}$ ) be a maximum density segment with length at least $\ell$ for $A_{1, i}$ (respectively, $A_{i, n}$ ). Clearly, $P_{i}$ and $Q_{i+1}$ are disjoint segments of $A$ for each $i=1,2, \ldots, n-1$. Chung and Lu's algorithm has the nice feature that can process the input sequence in an online manner. Therefore, all $P_{i}$ and $Q_{i}$ with $1 \leq i \leq n$ can be computed by Chung and Lu's algorithm in $O(n)$ time. The set $\left\{P_{i}, Q_{i+1}\right\}$ with maximum $D\left(\left\{P_{i}, Q_{i+1}\right\}\right)$ is clearly an optimal solution for the case with $k=2$. Therefore, we have the following theorem.

Theorem 2. It takes $O(n)$ time to compute a pair of disjoint segments of a length-n sequence, each has length at least $\ell$, such that the sum of their densities is maximized.

\section{Our Algorithm for $k=3$}

Suppose that $S_{o 1}, S_{o 2}$ and $S_{o 3}$ form an optimal set of segments for the case with $k=3$. We first find a maximum-density segment $S_{M}=A_{m_{i}, m_{j}}$ in $A$. We also compute maximum-density segments $S_{L}=A_{l_{i}, l_{j}}$ in $A_{1, m_{i}-1}$ and $S_{R}=A_{r_{i}, r_{j}}$ in $A_{m_{j}+1, n}$, respectively. Then we find the optimal two disjoint density segments $\left\{S_{L 1}, S_{L 2}\right\}$ in $A_{1, m_{i}-1}$ and $\left\{S_{R 1}, S_{R 2}\right\}$ in $A_{m_{j}+1, n}$. Let $\left\{S_{M^{\prime}}, S_{M^{\prime \prime}}\right\}$ be the element in

$$
\left\{\left\{S_{L}, S_{R}\right\},\left\{S_{L 1}, S_{L 2}\right\},\left\{S_{R 1}, S_{R 2}\right\}\right\}
$$

that has maximum sum of densities. Moreover, we find the maximum-density segment $S_{L L}=A_{l l_{i}, l l_{j}}$ in $A_{1, l_{i}-1}$ and the maximum-density segment $S_{R R}=$ $A_{r r_{i}, r r_{j}}$ in $A_{r_{j}+1, n}$. Furthermore, we find the maximum density segment $S_{L L L}$ in $A_{1, l l_{i}-1}$ and the maximum-density segment $S_{R R R}$ in $A_{r r_{j}+1, n}$. For brevity, we use $S_{x} \sim S_{y}$ (respectively, $S_{x} \leftrightarrow S_{y}$ ) to denote that segments $S_{x}$ and $S_{y}$ overlap (respectively, are disjoint). Let $U$ be the set of segments which are intersect to $S_{M}$ with length from $\ell$ to $2 \ell-1$. Finally, for each segment $S$ in $U$, we perform the following Algorithm 1 to find three disjoint segments $\left\{S_{1}, S_{2}, S_{3}\right\}$ with $\left\{S_{1}, S_{2}, S_{3}\right\} \cap S \neq \emptyset$. 


\section{Algorithm 1:}

1. For each segment $S_{v}=A_{v_{i}, v_{j}}$ in $U$, let $S_{2}=S_{v}$. do

1.1. (Case 1: $S_{\boldsymbol{v}} \sim \boldsymbol{a}_{\boldsymbol{m}_{i}}$ but $S_{\boldsymbol{v}} \leftrightarrow \boldsymbol{a}_{\boldsymbol{m}_{j}}$ ): Find the maximum-density segment $S_{R^{\prime}}$ in $A_{v_{j}+1, m_{j}+2 \ell-2}$. Then let $S_{3}=S_{R^{\prime}}$.

If $S_{v} \leftrightarrow S_{L}$ then $S_{1}=S_{L}$

else

If $S_{v} \sim S_{L}$ but $S_{v} \leftrightarrow S_{L L}$ then find the maximum-density segment

$S_{L^{\prime}}$ in $A_{l_{i}-2 \ell+2, v_{i}-1}$ then let $S_{1}$ be the maximum density segment between $S_{L^{\prime}}$ and $S_{L L}$.

else find the maximum-density segment $S_{L^{\prime}}$ in $A_{l l_{i}-2 \ell+2, v_{i}-1}$ then let

$S_{1}$ be the maximum density segment between $S_{L^{\prime}}$ and $S_{L L L}$.

1.2. (Case 2: $S_{v} \sim a_{m_{j}}$ but $S_{v} \leftrightarrow a_{m_{i}}$ ): Find the maximum-density segment $S_{L^{\prime \prime}}$ in $A_{m_{i}-2 \ell+2, v_{i}-1}$. Then let $S_{1}=S_{L^{\prime \prime}}$.

If $S_{v} \leftrightarrow S_{R}$ then let $S_{3}=S_{R}$

else

If $S_{v} \sim S_{R}$ but $S_{v} \leftrightarrow S_{R R}$ then find the maximum-density segment $S_{R^{\prime \prime}}$ in $A_{v_{j}+1, r_{j}+2 \ell-2}$ then let $S_{3}$ be the maximum density segment between $S_{R^{\prime \prime}}$ and $S_{R R}$.

else find the maximum-density segment $S_{R^{\prime \prime}}$ in $A_{v_{j}+1, r r_{j}+2 \ell-2}$ then let $S_{3}$ be the maximum density segment between $S_{R^{\prime \prime}}$ and $S_{R R R}$.

1.3. (Case 3: $\boldsymbol{S}_{\boldsymbol{v}} \subset \boldsymbol{S}_{\boldsymbol{m}}$ ): Find the maximum-density segments $S_{L^{\prime \prime \prime}}$ and end for $S_{R^{\prime \prime \prime}}$ in $A_{m_{i}-2 \ell+2, v_{i}-1}$ and $A_{v_{j}+1, m_{j}+2 \ell-2}$. Let $\left\{S_{1}, S_{3}\right\}=\left\{S_{L^{\prime \prime \prime}}, S_{R^{\prime \prime \prime}}\right\}$.

2. Let $\left\{S_{a}, S_{b}, S_{c}\right\}$ be the maximum total density segments in all these three disjoint segments $\left\{S_{1}, S_{2}, S_{3}\right\}$.

Finally, if

$$
D\left(\left\{S_{a}, S_{b}, S_{c}\right\}\right) \leq D\left(\left\{S_{M}, S_{M^{\prime}}, S_{M^{\prime \prime}}\right\}\right),
$$

then let $\left\{S_{o 1}, S_{o 2}, S_{o 3}\right\}$ be $\left\{S_{M^{\prime}}, S_{M}, S_{M^{\prime \prime}}\right\}$; otherwise, let $\left\{S_{o 1}, S_{o 2}, S_{o 3}\right\}$ be $\left\{S_{a}, S_{b}, S_{c}\right\}$. Though there are $O\left(\ell^{2}\right)$ iterations in Algorithm 1, we only need $O\left(\ell^{2}\right)$ time in total. We can pre-process to find all $S_{R^{\prime}}$ in case 1 , all $S_{R^{\prime \prime \prime}}$ in case 3 , all $S_{L^{\prime \prime}}$ in case 2 and all $S_{L^{\prime \prime \prime}}$ in case 3 in $O\left(\ell^{2}\right)$ time. Because the lengths of $A_{m_{i}-2 \ell+2, v_{i}-1}$ and $A_{v_{j}+1, m_{j}+2 \ell-2}$ are $O(\ell)$ and the length of $S_{M}$ is at most $2 \ell-1$. Also pre-process to find all $S_{L^{\prime}}$ in case 1 and all $S_{R^{\prime \prime}}$ in case 2 take $O\left(\ell^{2}\right)$ time. As a result, the time complexity of Algorithm 1 is $O\left(\ell^{2}\right)$.

Theorem 3. It takes $O\left(n+\ell^{2}\right)$ time to compute three disjoint segments of a length-n sequence, each has length at least $\ell$, such that the sum of their densities is maximized.

Proof. Since the time complexity of Algorithm 1 is $O\left(\ell^{2}\right)$, our algorithm runs in $O\left(n+\ell^{2}\right)$ time. It remains to prove the correctness of our algorithm. For any three disjoint segments $\left\{S_{1}, S_{2}, S_{3}\right\}$ in $A$, we will show

$$
D\left(\left\{S_{o 1}, S_{o 2}, S_{o 3}\right\}\right) \geq D\left(\left\{S_{1}, S_{2}, S_{3}\right\}\right) .
$$


For convenience, let $S_{1}$ be the left segment, let $S_{2}$ be the middle segment, and let $S_{3}$ be the right segment for the three disjoint segments $\left\{S_{1}, S_{2}, S_{3}\right\}$ in $A$. First, if each of $S_{1}, S_{2}$ and $S_{3}$ does not overlap with $S_{M}$, then

$$
D\left(\left\{S_{M}, S_{M^{\prime}}, S_{M^{\prime \prime}}\right\}\right) \geq D\left(\left\{S_{1}, S_{2}, S_{3}\right\}\right) .
$$

If only one segment of $\left\{S_{1}, S_{2}, S_{3}\right\}$ overlaps with $S_{M}$, then

$$
D\left(\left\{S_{M}, S_{M^{\prime}}, S_{M^{\prime \prime}}\right\}\right) \geq D\left(\left\{S_{1}, S_{2}, S_{3}\right\}\right) .
$$

Hence, the rest of the proof assumes that at least two segments of $\left\{S_{1}, S_{2}, S_{3}\right\}$ overlaps with $S_{M}$ and

$$
D\left(\left\{S_{1}, S_{2}, S_{3}\right\}\right)>D\left(\left\{S_{M^{\prime}}, S_{M}, S_{M^{\prime \prime}}\right\}\right) .
$$

Without loss of generality, we may assume that segment $S_{2}=S_{v}=A_{v_{i}, v_{j}}$ overlaps with $S_{M}$. Then we consider the following three cases. Case 1: $S_{v} \sim a_{m_{i}}$ but $S_{v} \leftrightarrow a_{m_{j}}$, case 2: $S_{v} \sim a_{m_{j}}$ but $S_{v} \leftrightarrow a_{m_{i}}$, and case 3: $S_{v} \subset S_{m}$. We prove the result for case 1 and case 3 . The case 2 can be shown similar to case 1. For case 1 , let $S_{R^{\prime}}$ is the maximum-density segment in $A_{v_{j}+1, m_{j}+2 \ell-2}$ and $S_{3}=S_{R^{\prime}}$. Because $d\left(S_{1}\right) \leq d\left(S_{L}\right)$ and $d\left(S_{2}\right) \leq d\left(S_{M}\right)$, the segment $S_{3}$ must be a subsequence in $A_{v_{j}+1, m_{j}+2 \ell-2}$; otherwise, we have

$$
D\left(\left\{S_{L}, S_{M}, S_{R}\right\}\right) \geq D\left(\left\{S_{1}, S_{2}, S_{3}\right\}\right) .
$$

Hence, we only choose a best $S_{1}$ in $A_{1, v_{i}-1}$. We consider the following three cases. (1) if $S_{v} \leftrightarrow S_{L}$, we only let $S_{1}=S_{L}$ because $S_{L}$ is the maximum-density segment in $A_{1, m_{i}-1}$. (2) If $S_{v} \sim S_{L}$ but $S_{v} \leftrightarrow S_{L L}$. For $S_{1}$, we only consider the segments $S_{L L}$ and $S_{L^{\prime}}$, where $S_{L^{\prime}}$ is a maximum-density segment in $A_{l_{i}-2 \ell+2, v_{i}-1}$. Because $S_{1} \sim S_{L}$, segment $S_{1}$ is either in $A_{1, l_{i}-1}$ or in $A_{l_{i}-2 \ell+2, v_{i}-1}$. (3) $S_{v} \sim S_{L}$ and $S_{L L}$. For $S_{1}$, we only consider the segments $S_{L L L}$ and $S_{L^{\prime}}$, where $S_{L^{\prime}}$ is a maximumdensity segment in $A_{l l_{i}-2 \ell+2, v_{i}-1}$. Because $S_{1} \sim S_{L L}$, segment $S_{1}$ is either in $A_{1, l l_{i}-1}$ or in $A_{l l_{i}-2 \ell+2, v_{i}-1}$. For case 3 , let $S_{L^{\prime \prime \prime}}$ is the maximum-density segment in $A_{m_{i}-2 \ell+2, v_{i}-1}$ and $S_{R^{\prime \prime \prime}}$ is the maximum-density segment in $A_{v_{j}+1, m_{j}+2 \ell-2}$. Because $d\left(S_{v}\right) \leq d\left(S_{M}\right)$, we only let $\left\{S_{1}, S_{2}, S_{3}\right\}=\left\{S_{L^{\prime \prime \prime}}, S_{v}, S_{R^{\prime \prime \prime}}\right\}$. Otherwise, we have

$$
D\left(\left\{S_{L}, S_{M}, S_{R}\right\}\right) \geq D\left(\left\{S_{1}, S_{2}, S_{3}\right\}\right)
$$

\section{Conclusion}

We have shown the first known polynomial-time algorithm to compute multiple disjoint segments whose sum of densities is maximized. An immediate open question is whether the problem can be solved in $o(n \ell k)$ time. Also, it would be interesting to see our techniques for $k=2,3$ to be generalized to the cases with larger $k$. 


\section{References}

1. P. Berman, P. Bertone, B. DasGupta, M. Gerstein, M.-Y. Kao and M. Snyder: Fast Optimal Genome Tiling with Applications to Microarray Design and Homology Search. Journal of Computational Biology, 11:766-785, 2004.

2. K.-M. Chung and H.-I. Lu: An Optimal Algorithm for the Maximum-Density Segment Problem. SIAM Journal on Computing, 34:373-387, 2004.

3. R. Durbin, S. Eddy, A. Krogh, and G. Mitchison: Biological Sequence Analysis. Cambridge University Press, 1998.

4. T.-H. Fan, S. Lee, H.-I. Lu, T.-S. Tsou, T.-C. Wang, A. Yao: An Optimal Algorithm for Maximum-Sum Segment and Its Application in Bioinformatics. In Proceedings of the 8th International Conference on Implementation and Application of Automata, Lecture Notes in Computer Science 2759, 251-257, Santa Barbara, July 2003, Springer-Verlag.

5. M. Gardiner-Garden, and M. Frommer: CpG Islands in Vertebrate Genomes. Journal of Molecular Biology, 196:261-282, 1987.

6. M.H. Goldwasser, M.-Y. Kao, and H.-I. Lu: Linear-Time Algorithms for Computing Maximum-Density Sequence Segments with Bioinformatics Applications. Journal of Computer and System Sciences, 70:128-144, 2005.

7. U. I. Gupta, D. T. Lee, and J. Y.-T. Leung: Efficient Algorithms for Interval Graphs and Circular-Arc Graphs, Networks 12:459-467, 1982.

8. J. Y. Hsiao, C. Y. Tang, and R. S. Chang: An Efficient Algorithm for Finding a Maximum Weight 2-Independent Set on Interval Graphs, Information Processing Letters, 43(5):229-235, 1992.

9. X. Huang: An algorithm for Identifying Regions of a DNA Sequence That Satisfy a Content Requirement. Computer Applications in the Biosciences, 10:219-225, 1994.

10. S. K. Kim: Linear-Time Algorithm for Finding a Maximum-Density Segment of a Sequence. Information Processing Letters, 86:339-342, 2003.

11. F. Larsen, R. Gundersen, R. Lopez, and H. Prydz: CpG Islands as Gene Marker in the Human Genome. Genomics, 13:1095-1107, 1992.

12. Y.-L. Lin, T. Jiang, K.-M. Chao: Efficient Algorithms for Locating the LengthConstrained Heaviest Segments with Applications to Biomolecular Sequence Analysis. Journal of Computer and System Sciences, 65:570-586, 2002.

13. Y.-L. Lin, X. Huang, T. Jiang, K.-M. Chao: MAVG: Locating Non-overlapping Maximum Average Segments in a Given Sequence. Bioinformatics, 19:151-152, 2003.

14. A. Nekrutenko and W.-H. Li: Assessment of Compositional Heterogeneity within and between Eukaryotic Genomes. Genome Research, 10:1986-1995, 2000.

15. P. Rice, I. Longden, and A. Bleasby: EMBOSS: The European Molecular Biology Open Software Suite. Trends in Genetics, 16:276-277, 2000.

16. P. H. Sellers: Pattern Recognition in Genetic Sequences by Mismatch Density. Bulletin of Mathematical Biology, 46:501-514, 1984.

17. N. Stojanovic, L. Florea, C. Riemer, D. Gumucio, J. Slightom, M. Goodman, W. Miller, and R. Hardison: Comparison of Five Methods for Finding Conserved Sequences in Multiple Alignments of Gene Regulatory Regions. Nucleic Acids Research, 27:3899-3910, 1999.

18. D. Takai, and P.A. Jones: Comprehensive Analysis of CpG Islands in Human Chromosomes 21 and 22. Proceedings of the National Academy of Sciences, 99:3740$3745,2002$. 